\title{
Photothermal excitation of microcantilevers in liquids
}

\author{
D. Ramos, J. Tamayo, ${ }^{\text {a) }}$ J. Mertens, and M. Calleja \\ BioNanoMechanics Laboratory, National Centre for Microelectronics, IMM-CNM, CSIC, \\ Isaac Newton 8 (PTM), Tres Cantos 28760, Madrid, Spain
}

(Received 3 October 2005; accepted 28 March 2006; published online 21 June 2006)

\begin{abstract}
We report the selective excitation of the flexural modes of microcantilevers in aqueous solutions, by applying the photothermal excitation technique. The experiments show that a particular vibration mode can be efficiently excited by focusing the intensity-modulated laser beam on regions of high curvature of the vibration shape. In addition, the resulting resonant peaks in liquid appear distorted by an amplitude component that decreases with the frequency. This distortion produces a shift of the resonance to lower frequencies. A theoretical model based on the transformation of optical energy into mechanical energy via an intermediate thermal stage is proposed to interpret the experimental results. The theory shows that the driven oscillation of the cantilever depends on the curvature of the eigenmode at the excitation position and the heating induced by the excitation laser, which decreases with the frequency. The results reported here set the basis for efficient excitation of high vibration modes in liquids and for optimized design of optically driven microresonators. (C) 2006 American Institute of Physics. [DOI: 10.1063/1.2205409]
\end{abstract}

\section{INTRODUCTION}

Resonating microcantilevers in liquids are the crucial elements in atomic force microscopy (AFM) and in sensor devices, for high resolution imaging of the native structure of biomolecules $^{1-3}$ and for sensitive biological detection. ${ }^{4-9}$ Further progress of these important applications requires to overcome the limitations inherent to operation in liquids. Firstly, resonant vibration of the cantilever is impeded by the large damping and added inertial mass of the liquid. ${ }^{10}$ Both effects, respectively, result into a low quality factor $(Q$ factor) and the shift of the resonance to lower frequencies, considerably degrading the sensitivity of the dynamic measurements. ${ }^{3}$ Secondly, liquid damping limits the amount of cantilever vibration, and therefore the signal-to-noise ratio. In addition, electrical excitation methods are technically hindered by the need for isolation of the actuators and driving circuits from the liquid.

Microcantilevers are often driven in liquid by either mechanical or magnetic excitation methods. Mechanical excitation is accomplished by a piezoelectric crystal near the cantilever base conveniently isolated from the liquid. However, spurious resonances from the fluid cell, cantilever chip, and fluid are also excited that make difficult to discern the resonance own to the cantilever. ${ }^{11}$ Magnetic excitation is achieved by coating the cantilevers with a thin ferromagnetic layer and applying a sinusoidal magnetic field. ${ }^{12}$ However, magnetic coatings are often corroded in liquid and can produce an intolerable cantilever bending.

The sensitivity in liquids can be enhanced by using higher vibration modes due to the higher resonance frequencies and higher quality factors. ${ }^{13}$ However, the driving techniques described above provide a uniform force over the cantilever limiting the vibration of high flexural modes. In

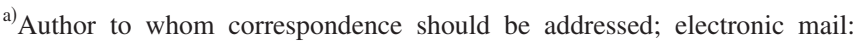
jtamayo@imm.cnm.csic.es
}

addition, magnetic excitation of cantilevers at high frequencies is hampered by the high impedance of the driving electronic circuit.

In this work, we apply the photothermal modulation technique for the selective excitation of flexural modes of a microcantilever in liquid. Although the photothermal excitation of microcantilevers has been presented in a number of papers, ${ }^{14-17}$ a consistent theoretical understanding is still lacking, and in addition, the effect of the photothermal excitation on the different flexural modes in liquid environments has not been studied. Here, we present experiments showing how the vibration of the first three vibration modes depends on the position of the intensity modulated laser beam along the cantilever. The experimental results are supported by a theoretical model.

\section{MATERIALS AND METHODS}

A schematic drawing of the experimental setup is shown in Fig. 1. The excitation of the cantilevers was achieved by a laser diode beam whose intensity can be modulated from dc up to $500 \mathrm{kHz}$ by using a transistor-transistor logic (TTL) modulation input $(5 \mathrm{~mW}, 635 \mathrm{~nm}$; Edmund Optics Ltd.). The laser beam was transversally focused on the cantilever with a spot size of about $10 \mu \mathrm{m}$ in diameter achieved by means of a convergent lens. The excitation laser diode was mounted on a microposition $X Y$ stage in order to control the position of the focused beam along the cantilever with a $\pm 10 \mu \mathrm{m}$ accuracy. Commercial silicon nitride cantilevers (Olympus) were used for the experiments. Their length, width, and thickness were 200,20 , and $0.8 \mu \mathrm{m}$, respectively. In order to enhance the photothermal effect, the original reflective gold coating of the cantilever was removed by immersion in a mixture of hydrochloric acid and nitric acid (3:1), and this cantilever side was coated with $5 \mathrm{~nm}$ of chromium and $70 \mathrm{~nm}$ of gold using thermal evaporation. The chromium layer was used to improve the adhesion between 


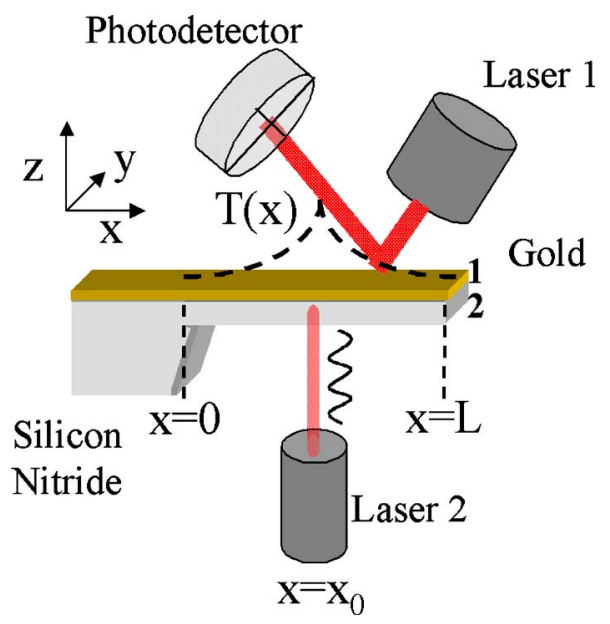

FIG. 1. (Color online) Schematic depiction of the experimental setup used for the photothermal excitation. The output intensity of the laser 2 is modulated for exciting the cantilever, whereas the laser 1 is used for measuring the cantilever vibration.

the gold and the silicon nitride. The cantilever vibration was measured by using the optical beam deflection technique, i.e., a nonmodulated laser diode beam $(3 \mathrm{~mW}, 635 \mathrm{~nm}$; Edmund Optics Ltd.) was focused on near the cantilever free end, and the reflected beam was collected into a four quadrant position sensitive detector (Hamamatsu). Since the output power of this laser is constant, it does not affect the dynamic response. The photocurrents of the position sensitive detector were preamplified and filtered by current-tovoltage converters (HMS). The amplified signals were then connected to a personal computer (PC) via analog-to-digital converters (National Instruments). A frequency generator (Agilent 33220A, $20 \mathrm{MHz}$ function) controlled by the PC via universal serial bus (USB) was used to modulate the intensity of the excitation laser. A square wave form was used to modulate the laser intensity, switching it on and off. Fast frequency sweeps in the order of $0.01 \mathrm{~s}$ were achieved to measure the resonant peaks, by synchronizing the frequency sweeping and the acquisition time during the experiment. Then, a fast Fourier transform of the vibration is performed to obtain the resonant peaks.

\section{EXPERIMENTAL RESULTS}

Measurements were performed in a homemade PolyMethylMethAcrylate (PMMA) microfluidic cell, in ultrapure and de-ionized water at room temperature. Figure 2 depicts the shape of the first three natural vibration modes for a singly clamped cantilever beam. The clamp and the cantilever free end are situated at $x=0$ and $x=L$, respectively.

Figure 3(a) shows the frequency spectra of the cantilever vibration in water corresponding to the first flexural mode for different focusing positions, $x_{0}$, of the intensity-modulated laser beam along the cantilever. The discontinuous line shows the frequency spectrum of the thermal noise for the first vibration mode of the cantilever. The resonant frequency is $5.66 \mathrm{kHz}$ and the quality factor is very low, of about 2.0 , due to the high viscous damping in liquids. Both parameters are deduced by fitting the thermal noise spectrum with the damped harmonic oscillator model. ${ }^{18}$ When the intensity-

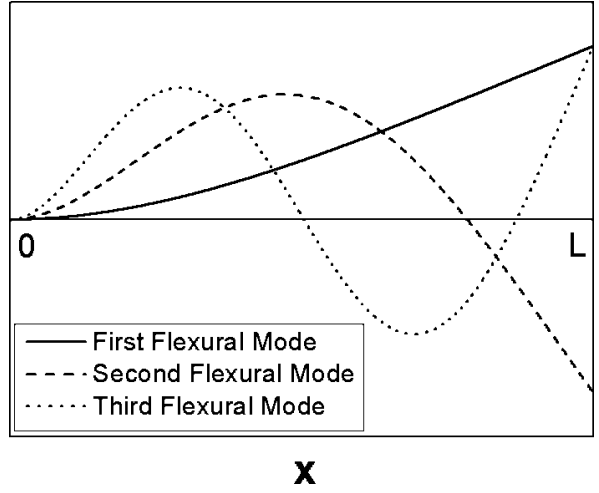

FIG. 2. Schematic depiction of the first three bending modes of a singly clamped cantilever. The clamping is placed at $x=0$.

modulated laser beam is focused on near the free cantilever end, $x_{0}=180 \mu \mathrm{m}$, no significant effect can be observed on the frequency spectrum. However, when the excitation laser is focused on the cantilever center, $x_{0}=100 \mu \mathrm{m}$, the amplitude at resonance increases about 125 times. The amplitude is further increased by displacing the excitation laser beam to $x_{0}=40 \mu \mathrm{m}$, but it falls when the laser is focused close to the clamp, at $x_{0}=20 \mu \mathrm{m}$. In addition, a symmetry loss in the optically driven resonance peak is observed, indicating that the driving force decreases with the frequency as can be deduced from the shape of the peaks. The frequencydependent driving force produces an effective resonance shift to lower frequencies, of about $5 \%-7 \%$ for $x_{0}$ equal to 20 and $40 \mu \mathrm{m}$, and in some cases, as for $x_{0}=100 \mu \mathrm{m}$, it makes
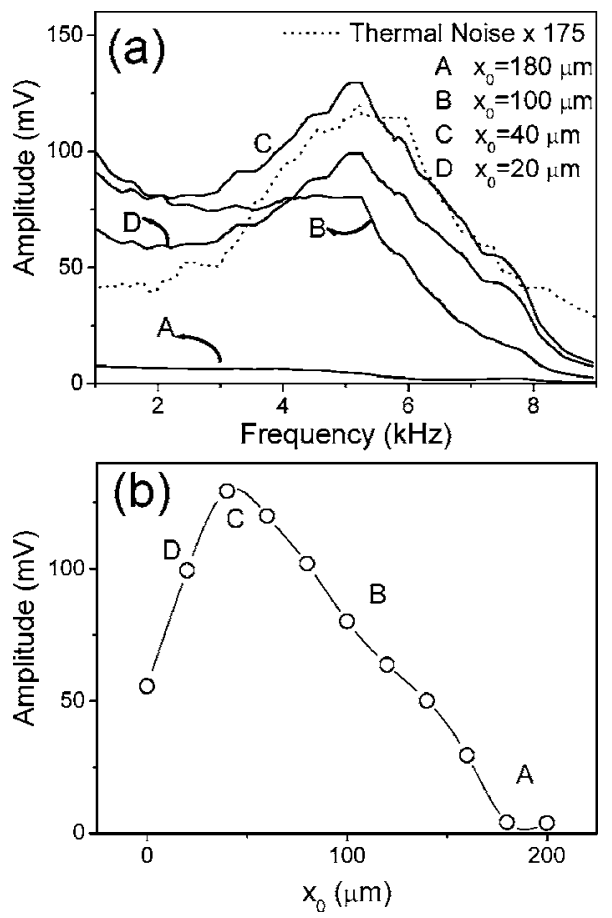

FIG. 3. Experimental results for the photothermal excitation of the first flexural mode for a cantilever immersed in water. (a) Frequency spectra for the cantilever vibration when the modulated laser is focused at different positions along the cantilever (continuous lines). The dashed line represents the thermal noise spectrum. (b) Amplitude at resonance as a function of the position of the intensity-modulated laser beam along the cantilever. The line between symbols is a guide to the eye only. 

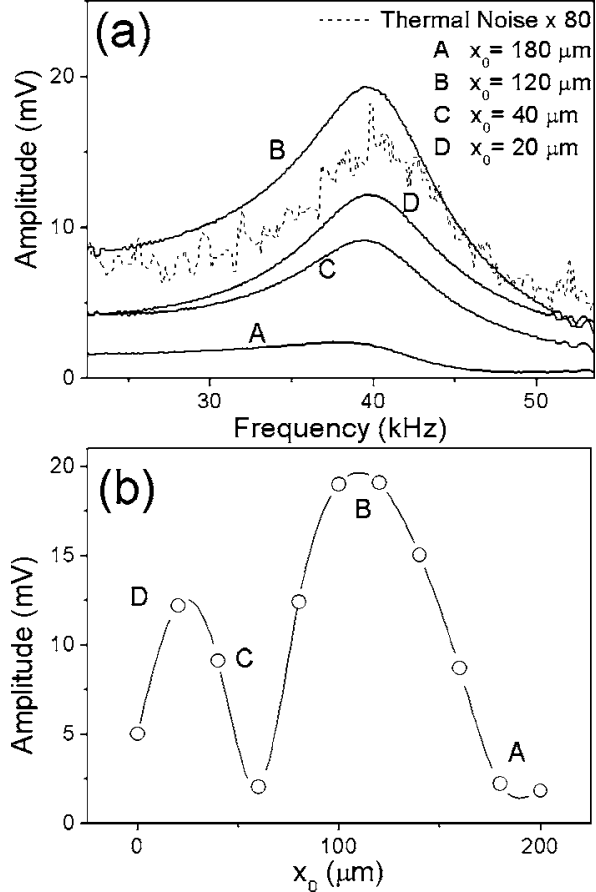

FIG. 4. Experimental results for the photothermal excitation of the second flexural mode for a cantilever immersed in water. (a) Frequency spectra for the cantilever vibration when the modulated laser is focused at different positions along the cantilever (continuous lines). The dashed line represents the thermal noise spectrum. (b) Amplitude at resonance as a function of the position of the intensity-modulated laser beam along the cantilever. The line between symbols is a guide to the eye only.

difficult to determine the resonant frequency clearly. The vibration amplitude at resonance as a function of $x_{0}$ is depicted in Fig. 3(b). The amplitude continuously decreases with the excitation position, $x_{0}$, from 40 to $180 \mu \mathrm{m}$.

Similarly, the frequency spectra of the second vibration mode is characterized as a function of $x_{0}$ (Fig. 4). The thermal noise spectrum [discontinuous line, Fig. 4(a)] shows the resonance at $39.67 \mathrm{kHz}$ with a quality factor of about 5.3 . The resonant frequency is in consistency with the EulerBernouilli beam theory ${ }^{18}$ that states a ratio between the resonant frequencies of the second and first vibration modes of about 6.27. A small shift of the resonant frequency to lower frequencies is observed. However, it does not noticeably affect the symmetry of the resonance curves as it occurs in the first vibration mode [continuous lines, Fig. 4(a)]. This frequency shift ranges between $0.7 \%$ and $1.0 \%$ and slightly depends on $x_{0}$. The amplitude at resonance depends largely on $x_{0}$ [Fig. 4(b)]. Two amplitude maxima are found at $x_{0}$ $=120 \mu \mathrm{m}$ and $x_{0}=20 \mu \mathrm{m}$ with three minima at $x_{0}=180 \mu \mathrm{m}$, $x_{0}=60 \mu \mathrm{m}$, and $x_{0}=0 \mu \mathrm{m}$.

Figure 5 shows the effect of the excitation by the intensity-modulated laser for the third vibration mode. The measured thermal noise [discontinuous line, Fig. 5(a)] reveals a resonance frequency of $114.96 \mathrm{kHz}$, in close agreement with the Euler-Bernoulli theory, and the quality factor is 8.0. Notice that the quality factor enhances with the index of the vibration mode. The resonance peaks are not significantly distorted by the optical excitation, and the shift of the resonance to lower frequencies is below $0.5 \%$ [continuous lines, Fig. 5(a)]. The amplitude at resonance versus the exci-
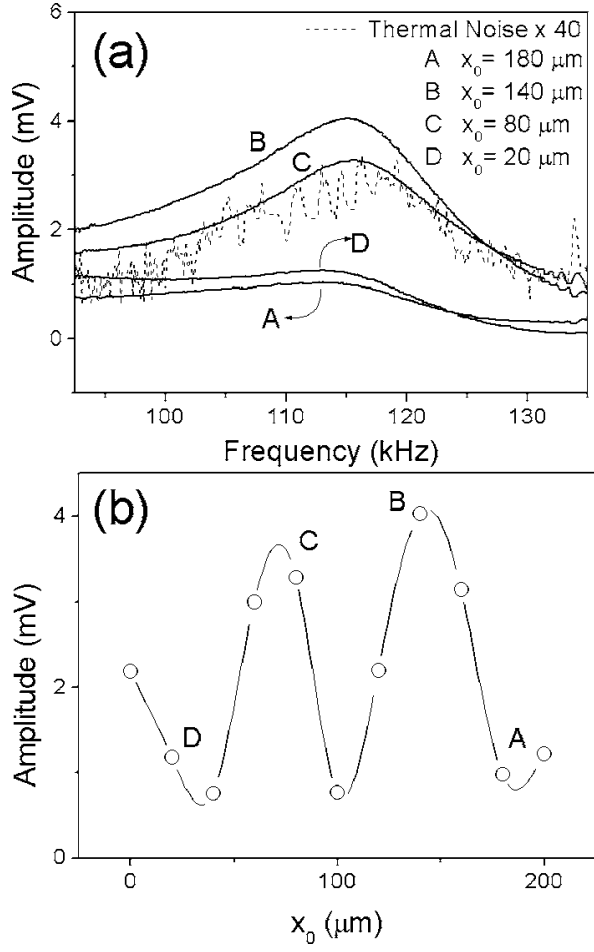

FIG. 5. Experimental results for the photothermal excitation of the third flexural mode for a cantilever immersed in water. (a) Frequency spectra for the cantilever vibration when the modulated laser is focused at different positions along the cantilever (continuous lines). The dashed line represents the thermal noise spectrum. (b) Amplitude at resonance as a function of the position of the intensity-modulated laser beam along the cantilever. The line between symbols is a guide to the eye only.

tation position, $x_{0}$, exhibits an oscillatory behavior with four local maxima located at the free end, the clamping, $x_{0}$ $=80 \mu \mathrm{m}$ and $x_{0}=140 \mu \mathrm{m}$ [Fig. 5(b)].

\section{THEORY}

Photothermal excitation is based on the transformation of optical energy into mechanical energy via an intermediate thermal stage. Thus, two phases can be distinguished in the optical activation that will be separately described in this section. First part is devoted to describe the temperature profile along the cantilever caused by the local periodic heating due to absorption of the optical energy. In second part, we describe the cantilever vibration due to the difference in the thermal expansion coefficients between the deposited metal film and the cantilever material.

\section{A. Photothermal effect}

The intensity-modulated light focused onto a region of the cantilever produces a time-dependent temperature distribution due to the absorption of optical energy. The absorbed power depends on the optical frequency of the excitation laser and the imaginary part of the index of refraction of the absorbing material. ${ }^{19}$ In our experimental conditions, assuming that the incident beam is perpendicular to the gold layer and the optical absorption by the water and PMMA fluid cell is negligible, we have calculated that the power absorbed by the cantilever is about $10 \%$ of the total incident power. As shown below, the thermal diffusion length is much larger 
than the cantilever thickness. Therefore, it is reasonable to assume that the temperature along the $z$ axis is constant. In addition, we can assume that the temperature along the $y$ axis is also constant as the laser spot diameter is comparable with the cantilever width. Therefore the time-dependent temperature distribution can be described by the one-dimensional heat diffusion equation, ${ }^{20}$

$$
\frac{\partial T}{\partial t}=K \frac{\partial^{2} T}{\partial x^{2}}-\beta T,
$$

where $K$ and $\beta$ are given by

$$
\begin{aligned}
& K=\frac{\kappa_{1} d_{1}+\kappa_{2} d_{2}}{c_{p 1} \rho_{1}+c_{p 2} \rho_{2}}, \\
& \beta=\frac{h_{1}+h_{2}}{c_{p 1} \rho_{1} d_{1}+c_{p 2} \rho_{2} d_{2}},
\end{aligned}
$$

where $\kappa$ is the thermal conductivity, $\rho$ the density, $c_{p}$ the specific heat capacity, $d$ the cantilever thickness, $h$ the heat transfer coefficient, and the subscripts 1 and 2 denote the metallic film and the cantilever material, respectively.

We assume that the temperature distribution oscillates at the same frequency as the light intensity, $\omega$, and then, we can write, $T(x, t)=T_{0}+\Delta T(x) e^{i \omega t}$, where $T_{0}$ is the temperature of the fluid that surrounds the cantilever. In addition, the temperature distribution must satisfy that the heat conducted outward from the cantilever end $x=L$ must be equal to that dissipated from the surface by free convection, $(\partial T / \partial x)_{x=L}$ $=-\left(h_{1} d_{1}+h_{2} d_{2}\right) /\left(\kappa_{1} d_{1}+\kappa_{2} d_{2}\right)\left(T_{x=L}-T_{0}\right)$. The solution for Eq. (1) that satisfies the above conditions is given by

$$
\begin{aligned}
& \Delta T(x)=\Delta T\left(x_{0}\right)\left\{\exp \left[-\left(\frac{1}{\delta_{\mathrm{th}}}+i \frac{2 \pi}{\lambda_{\mathrm{th}}}\right)\left(x-x_{0}\right)\right]\right. \\
& \left.+r \exp \left[\left(\frac{1}{\delta_{\mathrm{th}}}+i \frac{2 \pi}{\lambda_{\mathrm{th}}}\right)\left(x+x_{0}-2 L\right)+i \theta\right]\right\} \\
& x \geqslant x_{0}, \\
& \Delta T(x)=\Delta T\left(x_{0}\right)\left\{\exp \left[\left(\frac{1}{\delta_{\mathrm{th}}}+i \frac{2 \pi}{\lambda_{\mathrm{th}}}\right)\left(x-x_{0}\right)\right]\right. \\
& \left.\quad+r \exp \left[\left(\frac{1}{\delta_{\mathrm{th}}}+i \frac{2 \pi}{\lambda_{\mathrm{th}}}\right)\left(x+x_{0}-2 L\right)+i \theta\right]\right\} \\
& \quad x<x_{0},
\end{aligned}
$$

where $\delta_{\text {th }}$ and $\lambda_{\text {th }}$ are the thermal diffusion length and the thermal wavelength, respectively. The temperature distribution behaves as a damped oscillation, in which the temperature amplitude exponentially decays with the distance to the excitation position with a decay constant given by the thermal diffusion length, and the spatial period is given by the thermal wavelength. Both parameters depend on the thermal properties of the cantilever materials and on the frequency,

$$
\begin{aligned}
& \delta_{\mathrm{th}}=\left[\frac{2 K}{\beta+\left(\beta^{2}+\omega^{2}\right)^{1 / 2}}\right]^{1 / 2}, \\
& \lambda_{\mathrm{th}}=2 \pi\left[\frac{2 K}{-\beta+\left(\beta^{2}+\omega^{2}\right)^{1 / 2}}\right]^{1 / 2} .
\end{aligned}
$$
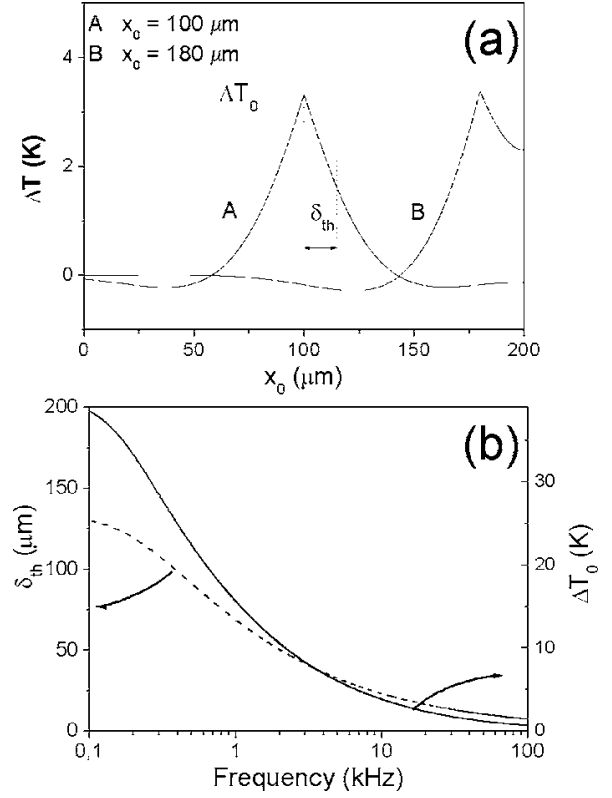

FIG. 6. (a) Theoretical simulation of the temperature profile for a 200- $\mu$ m-long cantilever when the intensity modulated laser is focused at $x_{0}=100 \mu \mathrm{m}$ and at $x_{0}=180 \mu \mathrm{m}$. (b) Simulated curves for the thermal diffusion length and the temperature raise at the illumination position as a function of the frequency.

The temperature distribution written in Eq. (4) is split up into two terms. The first summand represents the propagating thermal wave from the illuminated region, supposed as punctual $\left(x=x_{0}\right)$, towards the free end $\left(x \geqslant x_{0}\right)$ and the clamped end $\left(x<x_{0}\right)$. The second summand represents the reflected thermal wave at the cantilever free end. The parameters of the reflected thermal wave, $r$ and $\theta$, depend on the thermal properties of the materials, the heat transfer coefficient between the cantilever and the fluid and on the frequency of the intensity-modulated light. Both parameters are described in Appendix A, however, for the materials, fluids and frequencies commonly used for photothermal excitation of cantilevers $r \cong 1$ and $\theta \cong 0$. On the other hand, the amplitude of the propagating thermal waves, $\Delta T_{0}$, is deduced in Appendix B by making a heat balance at $x=x_{0}$. It is proportional to the absorbed light power and depends on the thermal properties of the material, the fluid and the frequency of the light modulation.

In order to simulate the experimental results shown above, the parameters used for the calculations are as follows. ${ }^{15}$ Subscripts 1 and 2 refer to gold and silicon nitride, respectively, $c_{p 1}=135 \mathrm{~J} \mathrm{Kg}^{-1} \mathrm{~K}^{-1}, c_{p 2}=750 \mathrm{~J} \mathrm{Kg}^{-1} \mathrm{~K}^{-1}, \rho_{1}$ $=19300 \mathrm{~K} \mathrm{gm}^{-3}, \rho_{2}=3400 \mathrm{~K} \mathrm{gm}^{-3}, \kappa_{1}=346 \mathrm{Wm}^{-1} \mathrm{~K}^{-1}$, and $\kappa_{2}=32 \mathrm{Wm}^{-1} \mathrm{~K}^{-1}$; the thickness of the gold film and silicon nitride cantilever are 70 and $800 \mathrm{~nm}$, respectively, the cantilever width $(W)$ and length $(L)$ are 20 and $200 \mu \mathrm{m}$, respectively; the heat transfer coefficient has been chosen from measurements of free heat convection between water and solids, $h_{1}=h_{2}=890 \mathrm{Wm}^{-2} \mathrm{~K}^{-1}$; and the absorbed light power amplitude is $0.5 \mathrm{~mW}$.

Figure 6(a) shows the temperature profile, $\Delta T(x)$, when the cantilever is illuminated by the excitation laser on the middle and near the free end. The intensity modulation frequency is $10 \mathrm{kHz}$. The temperature profile exhibits a maxi- 
mum at the illumination position that exponentially decays with the distance. The decay length is given by the thermal diffusion length, $\delta_{\text {th }}$. The effect of the reflected thermal waves is observed in the temperature profile when the excitation laser is focused near the free cantilever end. The temperature decay is smaller towards the cantilever free end than towards the clamp. The important role of the reflected thermal waves is due to the relatively low heat transmission at the cantilever/water interface and the higher constructive interference between the incident and reflected waves. On the other hand, both, the increase of temperature at the illumination position $\left(\Delta \mathrm{T}_{0}\right)$ and the thermal diffusion length $\left(\delta_{\mathrm{th}}\right)$ strongly decrease as the modulation frequency of the light intensity increases [Fig. 6(b)]. Thus, from 1 to $100 \mathrm{kHz}, \Delta T_{0}$ and $\delta_{\text {th }}$ decrease from $16 \mathrm{~K}$ and $68 \mu \mathrm{m}$ to $0.7 \mathrm{~K}$ and $7 \mu \mathrm{m}$, respectively. Therefore, higher modulation frequencies allow a confinement of the generated heat to smaller regions of the cantilever, but limit the amount of heat generated.

\section{B. Photothermal-assisted cantilever vibration}

The amplitude of the steady oscillation of a cantilever beam subject to a time-periodic force per unit length $f(x) e^{i \omega t}$ is described by the Euler-Bernoulli theory ${ }^{18}$

$$
A_{n}(\omega)=\frac{\psi_{n}^{0}(x)}{m\left[\omega_{n}^{2}-\omega^{2}+i\left(\omega_{n} \omega / Q_{n}\right)\right]} F_{n}
$$

where the subscript $n$ denotes the vibration mode, $m$ is the cantilever mass, $\omega_{n}$ the mode-dependent resonant frequency, $\psi_{n}{ }^{0}$ represents the normalized eigenfunctions that give the vibration mode shapes and $Q_{n}$ is the mode-dependent quality factor. The eigenfunctions and resonant frequency for a singly clamped beam are given ${ }^{21}$

$$
\begin{aligned}
& \psi_{n}^{0}(x)= A_{n}\left[\sin k_{n} x-\sinh k_{n} x\right. \\
&\left.+\frac{\left(\sin k_{n} L+\sinh k_{n} L\right)\left(\cosh k_{n} x-\cos k_{n} x\right)}{\cos k_{n} L+\cosh k_{n} L}\right], \\
& \omega_{n}=2 \pi f_{n} \cong k_{n}^{2} \frac{d}{L^{2}} \sqrt{\frac{E_{\text {eff }}}{12 \rho_{\text {eff }}}},
\end{aligned}
$$

where $d=d_{1}+d_{2}$ is the thickness. $E_{\text {eff }}$ and $\rho_{\text {eff }}$ are the effective Young's modulus and cantilever density, respectively, and are given by ${ }^{22}$

$$
E_{\text {eff }}=\frac{E_{1}{ }^{2} r_{1}{ }^{4}+E_{2}{ }^{2} r_{2}{ }^{4}+2 E_{1} E_{2} r_{1} r_{2}\left(2 r_{1}{ }^{2}+2 r_{2}{ }^{2}+3 r_{1} r_{2}\right)}{E_{1} r_{1}+E_{2} r_{2}},
$$

$$
\rho_{\mathrm{eff}}=\rho_{1} r_{1}+\rho_{2} r_{2},
$$

where $r_{1}=d_{1} / d$ and $r_{2}=d_{2} / d$.

$F_{n}$ represents the effective force acting on the cantilever that can be calculated from the projection of the excitation force per unit length on the corresponding vibration-mode shape,

$$
F_{n}=\int_{0}^{L} f(x) \psi_{n}^{0}(x) d x
$$

The heat generated by the laser beam produces a differential longitudinal stress across the cantilever thickness due to the difference in the thermal expansion coefficients of the cantilever materials. The longitudinal stress is given by $\alpha_{1} E_{1} \Delta T(x) e^{i \omega t}$ for $z>0$ and $\alpha_{2} E_{2} \Delta T(x) \mathrm{e}^{i \omega t}$ for $z<0 \quad(z=0$ is the gold and silicon nitride interface); where $\alpha$ is the thermal expansion coefficient. The differential longitudinal stress produces a bending moment that can be assumed to be constant over the length of infinitesimal elements. ${ }^{23}$ Hence, a temperature variation at the length element between $x_{0}$ and $x_{0}+d x_{0}$ causes a bending moment distribution $d M_{\mathrm{th}}(x) e^{i \omega t}$, where,

$$
\begin{aligned}
d M_{\mathrm{th}}(x) \cong & W\left[\int_{0}^{d_{1}} \alpha_{1} E_{1}\left(z-z_{0}\right) d z+\int_{-d_{2}}^{0} \alpha_{2} E_{2}\left(z-z_{0}\right) d z\right] \\
& \times\left[H\left(x-x_{0}-d x_{0}\right)-H\left(x-x_{0}\right)\right] \Delta T\left(x_{0}\right),
\end{aligned}
$$

where $W$ is the cantilever width, $z_{0}$ is the position of the neutral axis with respect to the bimaterial interface, and $H$ represents the Heaviside step function. The factor $\left[H\left(x-x_{0}\right.\right.$ $\left.\left.-d x_{0}\right)-H\left(x-x_{0}\right)\right]$ can be approximately written as $\delta(x$ $\left.-x_{0}\right) d x_{0}$, where $\delta$ represents the Dirac's delta function, whereas the neutral axis is determined by imposing that the net longitudinal force in the cantilever must be zero,

$$
z_{0}=\frac{1}{2} \frac{E_{1} d_{1}^{2}-E_{2} d_{2}^{2}}{E_{1} d_{1}-E_{2} d_{2}} \text {. }
$$

The bending moment distribution for a temperature profile along the cantilever is then given by $M_{\mathrm{th}}(x) e^{i \omega t}$, where the spatial part is obtained by integrating Eq. (13),

$$
\begin{aligned}
& M_{\mathrm{th}}(x)=-c_{\mathrm{th}} \int_{0}^{L} \Delta T\left(x_{0}\right) \delta\left(x-x_{0}\right) d x_{0}, \\
& \text { where } c_{\mathrm{th}}=W\left[\alpha_{1} E_{1} d_{1}\left(\frac{d_{1}}{2}-z_{0}\right)-\alpha_{2} E_{2} d_{2}\left(z_{0}-\frac{d_{2}}{2}\right)\right] .
\end{aligned}
$$

By applying the Euler-Bernoulli beam theory, the photothermal-induced bending moment is balanced by a transversal force whose spatial distribution per unit length is given by ${ }^{18}$

$$
f_{\mathrm{th}}(x)=-\frac{\partial^{2} M_{\mathrm{th}}(x, t)}{\partial x^{2}}
$$

Therefore, the effective force per unit length acting on the cantilever can be written as

$$
F_{n}=c_{\text {th }} \int_{0}^{L} \Delta T\left(x_{0}\right) d x_{0} \int_{0}^{L} \psi_{n}^{0}(x) \frac{\partial^{2}}{\partial x^{2}} \delta\left(x-x_{0}\right) d x .
$$

Using the properties of the Dirac's delta, the modedependent effective force can be written as

$$
F_{n}=c_{\text {th }} \int_{0}^{L} \Delta T\left(x_{0}\right) \frac{d^{2} \psi_{n}^{0}\left(x_{0}\right)}{d x_{0}{ }^{2}} d x_{0} .
$$



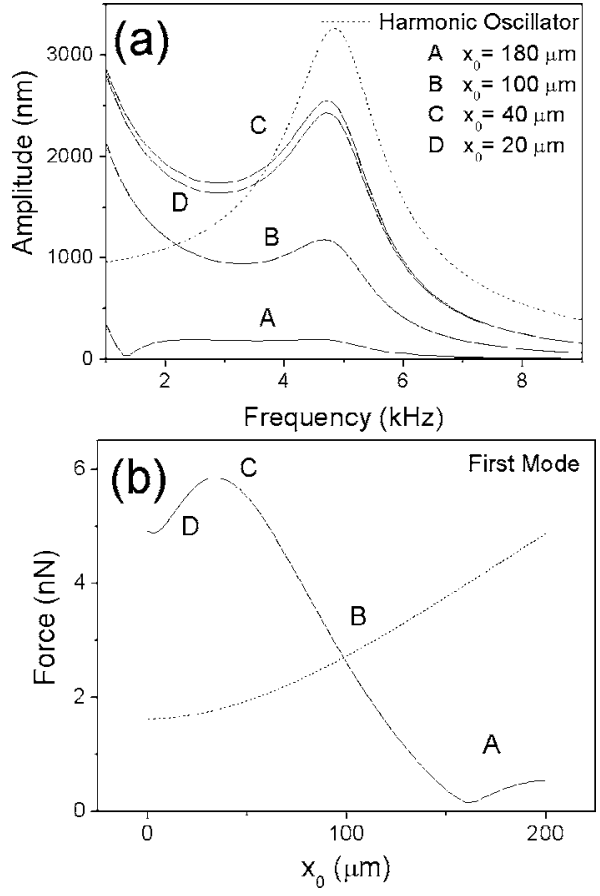

FIG. 7. Theoretical results for the photothermal excitation of the first flexural mode for a cantilever immersed in water. (a) Frequency spectra for the cantilever vibration when the modulated laser is focused at different positions along the cantilever length (continuous lines). The dashed line represents the result of the harmonic oscillator approximation. (b) Amplitude of the resulting excitation force as a function of the position where the intensity-modulated laser beam is focused along the cantilever. The dashed line represents the shape of the resonant mode.

\section{Theoretical results}

Figures 7-9 show the theoretical curves of the resonance for different excitation positions along the cantilever $\left(x_{0}\right)$, and the force generated by the photothermal excitation versus $x_{0}$, for the first three vibration modes. The parameters used to simulate the experimental results are those indicated in Sec. IV A plus the thermal expansion coefficients for the gold and silicon nitride, ${ }^{15} \alpha_{1}=14.2 \times 10^{-6}$ and $\alpha_{2}=3.2$ $\times 10^{-6}$. The resonant frequencies and the quality factors used in the calculations are those experimentally measured (see Sec. III); and the vibration amplitude was calculated at $190 \mu \mathrm{m}$ from the clamp, where the laser beam used in the optical detection technique was focused. The theoretical curves show the main features found in the experiments. Firstly, the vibration amplitude versus the excitation position follows a monotonic dependence for the first mode and a nonmonotonic dependence for the second and third modes. Secondly, the resonance peaks are a result of the product of the harmonic cantilever response and the optical excitation force, which decays with the frequency.

In general, as follows from Eq. (18), the excitation force is approximately proportional to the curvature of the eigenmode $\left(d^{2} \psi_{n}^{0} / d x^{2}\right)$ at the point where the intensity-modulated laser beam is focused. For the first vibration mode, the curvature of the vibration shape continuously increases from the free end to the clamped end. Thus the first vibration mode is more efficiently excited near the clamping region. The second and third vibration modes exhibit one and two vibration antinodes (points of amplitude maxima and therefore high
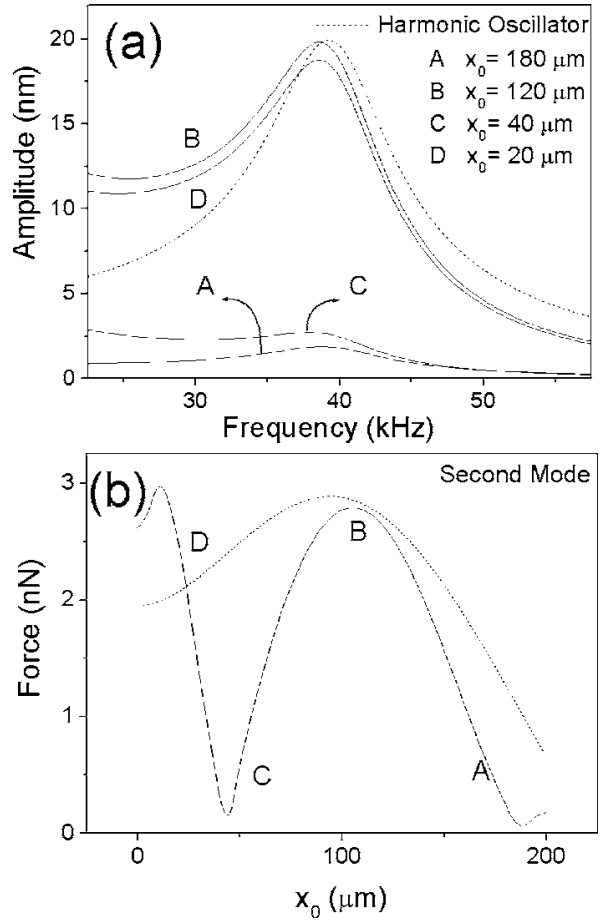

FIG. 8. Theoretical results for the photothermal excitation of the second flexural mode for a cantilever immersed in water. (a) Frequency spectra for the cantilever vibration when the modulated laser is focused at different positions along the cantilever length (continuous lines). The dashed line represents the result of the harmonic oscillator approximation. (b) Amplitude of the resulting excitation force as a function of the position where the intensity-modulated laser beam is focused along the cantilever. The dashed line represents the shape of the resonant mode.

curvature), respectively. Thus, these vibration modes are efficiently excited by focusing the intensity modulated laser close to the antinodes in addition to near the clamping region.

Interestingly, the optical excitation force exhibits a local maximum at the free end of the cantilever for the three vibration modes [Figs. 7(b), 8(b), and 9(b)]. This is also observed or inferred in the experimental results [Figs. 3(b), 4(b), and 5(b)]. This amplitude increase is caused by the reflected thermal waves, whose amplitude is significant when the excitation laser is focused near the free cantilever end. The reflected thermal waves arise from the low heat transmission between the cantilever and the water. Although the reflected thermal waves do not play a relatively important role for the 200- $\mu \mathrm{m}$-long cantilevers used here, these can produce significant effects on cantilevers with lengths similar or below the thermal diffusion length.

The heat diffusion critically depends on the frequency of the modulated laser. The higher the frequency, the smaller and more spatially confined the increase of the temperature [Fig. 6(b)]. Since, the excitation force is approximately proportional to the temperature, the frequency dependent increase of temperature changes the resonance shape and shifts the resonance to lower frequencies. This effect is more clearly seen in the first vibration mode, and is less noticeable as the vibration mode increases because of the smoother frequency dependence of the temperature increase at higher frequencies. In addition, the frequency dependency of the tem- 

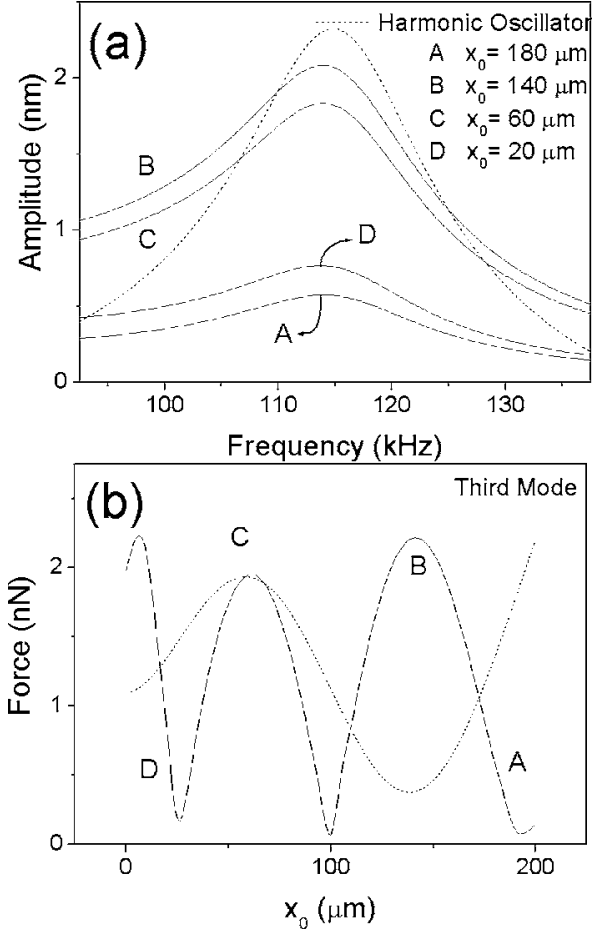

FIG. 9. Theoretical results for the photothermal excitation of the third flexural mode for a cantilever immersed in water. (a) Frequency spectra for the cantilever vibration when the modulated laser is focused at different positions along the cantilever length (continuous lines). The dashed line represents the result of the harmonic oscillator approximation. (b) Amplitude of the resulting excitation force as a function of the position where the intensity-modulated laser beam is focused along the cantilever. The dashed line represents the shape of the resonant mode.

perature increase slightly depends on the excitation position due to the reflected thermal waves. The consequence of this effect is twofold. Firstly, the effective shift of the resonance to lower frequencies slightly depends on the excitation position. Secondly, there is a small deviation of the excitation positions where amplitude maxima occur with respect to the position of the antinodes.

The vibration mode dependency of the excitation force is an interplay between the temperature increase that decays with the frequency, and the curvature of the vibration shape. Thus, the increase of temperature at resonance is of $15 \mathrm{~K}$ for the first vibration mode, whereas it is only of about $0.8 \mathrm{~K}$ for the third vibration mode. However, the maximal excitation force only decreases from $9.34 \mathrm{nN}$ for the first mode to $4.24 \mathrm{nN}$ for the third mode due to the increase of curvature of the vibration shape at the antinodes with the vibration mode index. Moreover, the smaller distance between antinodes as the mode index increases is also compensated by the major spatial confinement of the heat with the frequency. Thus, the thermal diffusion length at the resonant frequency of the first mode is of about $32.7 \mu \mathrm{m}$, whereas it is $7.3 \mu \mathrm{m}$ for the third mode, smaller than the distance between antinodes.

\section{CONCLUSIONS}

Here, we have reported the use of photothermal excitation for selective and efficient excitation of the different flexural modes of a microcantilever immersed in liquid. The ex- perimental results are supported by a theoretical model that comprises two mechanisms for the optical activation. The first mechanism is based on the heat transfer theory and deals with the transformation of the modulated optical power into a time-periodic increase of the local temperature of the cantilever. Second mechanism consists in the transformation of the local periodic heating into cantilever vibration via the bimetallic effect. Theory is consistent with the experimental results. Firstly, the excitation force for a given vibration mode is maximized when the cantilever is optically excited at regions with maxima of the curvature of the vibration shape. Secondly, the excitation force decreases with the frequency of the modulated excitation laser. This produces a distortion and a shift to lower frequencies of the resonance peaks that is more pronounced at low frequencies. Thirdly, the reflected thermal waves play an important role for separations between the excitation position and the cantilever free end smaller than the thermal diffusion length.

The photothermal excitation technique provides a noncontact and pointlike method for excitation of microcantilevers in liquid. Pointlike excitation methods allow easy identification of the cantilever resonance without the presence of spurious resonances as it occurs in the widely extended mechanoacoustic excitation methods. It only requires the presence of a metallic coating, that it is often used for reflectivity enhancement when optical detection is used. In addition, it provides an efficient way for excitation of high vibration modes, which offer higher quality factors and higher resonant frequencies, enhancing the sensitivity of the dynamic measurements in AFM in liquids and cantilever-based biosensors.

\section{ACKNOWLEDGMENTS}

The authors would like to acknowledge M. Álvarez for her assistance in the experiments. One of the authors (D.R.) would also like to acknowledge the fellowship funded by the Autonomic Community of Madrid. This work was supported by Spanish Ministry of Science (GEN2001-4856-C13-11).

\section{APPENDIX A}

The amplitude and phase of the reflecting waves [Eq. (4)] are calculated by applying the Newton's cooling law that produces the boundary condition ${ }^{21} \partial T /\left.\partial x\right|_{x=L}=-h / \lambda_{\mathrm{th}} T$,

$$
\begin{aligned}
r e^{i \theta}= & \exp \left\{-2 i\left[\frac{2 \pi}{\lambda_{\mathrm{th}}}\left(L-x_{0}\right)\right]\right\} \\
& \times\left\{\frac{-h / \lambda_{\mathrm{th}}+\left[1 / \delta_{\mathrm{th}}+i\left(2 \pi / \lambda_{\mathrm{th}}\right)\right]}{\left[1 / \delta_{\mathrm{th}}+i\left(2 \pi / \lambda_{\mathrm{th}}\right)\right]+\frac{h}{\lambda_{\mathrm{th}}}}\right\} .
\end{aligned}
$$

By separating the imaginary and real parts in Eq. (A1), we obtain

$$
\theta=\arctan \left[\frac{4 \pi h / \lambda_{\mathrm{th}}{ }^{2}}{\left(1 / \delta_{\mathrm{th}}\right)^{2}-\left(h / \lambda_{\mathrm{th}}\right)^{2}+\left(2 \pi / \lambda_{\mathrm{th}}\right)^{2}}\right],
$$




$$
r=\frac{\left\{\left[\left(1 / \delta_{\mathrm{th}}\right)^{2}-\left(h / \lambda_{\mathrm{th}}\right)^{2}+\left(2 \pi / \lambda_{\mathrm{th}}\right)^{2}\right]^{2}+\left(\frac{4 \pi h}{\lambda_{\mathrm{th}}^{2}}\right)^{2}\right\}^{1 / 2}}{\left(1 / \delta_{\mathrm{th}}+h / \lambda_{\mathrm{th}}\right)^{2}+\left(2 \pi / \lambda_{\mathrm{th}}\right)^{2}} .
$$

\section{APPENDIX B}

We calculate the amplitude of the thermal wave along the cantilever, $\Delta T_{0}$, [Eq. (4)] by making a heat balance at the illumination position $x=x_{0}$. The optical power absorbed by the cantilever must be equal to the heat loss. There are three mechanisms of heat loss: heat lost by conduction, the heat absorbed by the cantilever material, and the heat loss by the surface. The heat lost by conduction per unit time is

$$
P_{1}=-W\left(\kappa_{1} d_{1}+\kappa_{2} d_{2}\right)\left[\left.\frac{\partial T}{\partial x}\right|_{x=x_{0}{ }^{+}}-\left.\frac{\partial T}{\partial x}\right|_{x=x_{0}}\right] .
$$

The heat absorbed per unit time can be written as

$$
P_{2}=\left.W\left(c_{p 1} \rho_{1} d_{1}+c_{p 2} \rho_{2} d_{2}\right) \frac{\partial T}{\partial x}\right|_{x=x_{0}} \Delta x,
$$

where $\Delta x$ accounts for the diameter of the modulated laser beam on the cantilever. Finally the heat loss at the surface per unit time is

$$
P_{3}=W\left(h_{1}+h_{2}\right) T\left(x=x_{0}\right) \Delta x .
$$

The absorbed optical power, $P_{0} \cos (\omega t)$, must be equal to the total energy loss per unit time,

$$
\begin{aligned}
P_{0} \cos (\omega t)= & W \Delta T_{0}\left(\operatorname { c o s } ( \omega t ) \left\{\frac{2}{\delta_{\mathrm{th}}}\left(\kappa_{1} d_{1}+\kappa_{2} d_{2}\right)+\left(h_{1}+h_{2}\right) \Delta x\left[1+r \exp \left(-2 \frac{x_{0}}{\delta_{\mathrm{th}}}\right) \cos \gamma\right]-\left(c_{p 1} \rho_{1} d_{1}+c_{p 2} \rho_{2} d_{2}\right) \Delta x \omega r\right.\right. \\
& \left.\times \exp \left(-2 \frac{x_{0}}{\delta_{\mathrm{th}}}\right) \sin \gamma\right\}-\sin (\omega t)\left\{\frac{4 \pi}{\lambda_{\mathrm{th}}}\left(\kappa_{1} d_{1}+\kappa_{2} d_{2}\right)+\left(h_{1}+h_{2}\right) \Delta x \exp \left(-2 \frac{x_{0}}{\delta_{\mathrm{th}}}\right) \sin \gamma+\left(c_{p 1} \rho_{1} d_{1}+c_{p 2} \rho_{2} d_{2}\right) \Delta x \omega\right. \\
& \left.\left.\times\left[1+r \exp \left(-2 \frac{x_{0}}{\delta_{\mathrm{th}}}\right) \cos \gamma\right]\right\}\right),
\end{aligned}
$$

where, $\gamma=\theta-4 \pi x_{0} / \lambda_{\mathrm{th}}$. Therefore, the amplitude of the thermal wave can be written as

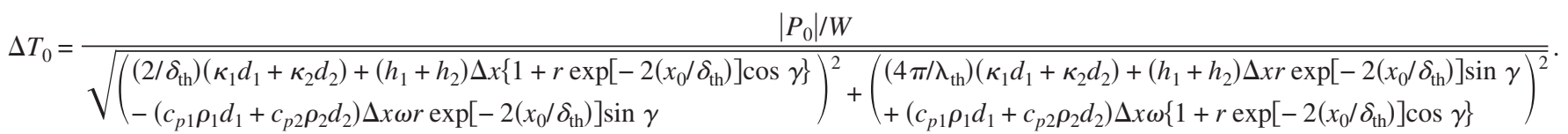

${ }^{1}$ A. Raab, W. Han, D. Badt, S. J. Smith-Gill, S. M. Lindsay, H. Schindler, and P. Hinterdorfer, Nat. Biotechnol. 17, 902 (1999).

${ }^{2}$ S. I. Lee, S. W. Howell, A. Raman, and R. Reifenberger, Phys. Rev. B 66, 115409 (2002).

${ }^{3}$ J. Tamayo, A. D. Humphris, R. J. Owen, and M. J. Miles, Biophys. J. 81, $526(2001)$.

${ }^{4}$ B. Illic, D. Czaplewski, H. G. Craighead, P. Neuzil, C. Campagnolo, and C. Batt, Appl. Phys. Lett. 77, 450 (2000).

${ }^{5}$ B. Illic, Y. Yang, and H. Craighead, Appl. Phys. Lett. 85, 2604 (2004).

${ }^{6}$ A. Gupta, D. Akin, and R. Bashir, Appl. Phys. Lett. 84, 1976 (2004).

${ }^{7}$ K. Y. Gfeller, N. Nugaeva, and M. Hegner, Biosens. Bioelectron. 21, 528 (2005).

${ }^{8}$ J. Tamayo, A. D. L. Humphris, A. M. Malloy, and M. J. Miles, Ultramicroscopy 86, 167 (2001).

${ }^{9}$ K. L. Ekinci, X. M. H. Huang, and M. L. Roukes, Appl. Phys. Lett. 84, 4469 (2004).

${ }^{10}$ G. Y. Chen, R. J. Warmack, A. Huang, and T. Thundat, J. Appl. Phys. 78, 1465 (1995).

${ }^{11}$ T. E. Schäffer, J. P. Cleveland, F. Ohnesorge, D. A. Walters, and P. K. Hansma, J. Appl. Phys. 80, 3622 (1996).
${ }^{12}$ W. Han, S. M. Lindsay, and T. Jing, Appl. Phys. Lett. 69, 4111 (1996).

${ }^{13}$ S. Dohn, R. Sandberg, W. Svendsen, and A. Boisen, Appl. Phys. Lett. 86, 233501 (2005)

${ }^{14}$ B. Illic, S. Krylov, K. Aubin, R. Reichenbach, and H. G. Craighead, Appl. Phys. Lett. 86, 193114 (2005).

${ }^{15}$ G. C. Ratcliff, D. A. Erie, and R. Superfine, Appl. Phys. Lett. 72, 1911 (1998).

${ }^{16}$ N. V. Lavrik and P. G. Datskos, Appl. Phys. Lett. 82, 2697 (2003).

${ }^{17}$ O. Marti, A. Ruf, M. Hipp, H. Bielefeldt, J. Colchero, and J. Mlynek, Ultramicroscopy 42, 345 (1992).

${ }^{18}$ S. Rast, C. Wattinger, U. Gysin, and E. Meyer, Rev. Sci. Instrum. 71, 2772 (2000)

${ }^{19}$ M. Shubert, Phys. Rev. B 53, 4265 (1996).

${ }^{20} \mathrm{H}$. Gröber, S. Erk, U. Grigull, Die Grundgesetze der Wärmeübertragung, 3rd ed. (Springer-Verlag, Berlin-Göttingen-Heidelberg, 1954).

${ }^{21}$ R. Sandberg, W. Svendsen, K. Molhave, and A. Boisen, J. Micromech. Microeng. 15, 1454 (2005).

${ }^{22}$ J. W. Yi, W. Y. Shih, and W.-H. Shih, J. Appl. Phys. 91, 1680 (2002).

${ }^{23}$ S. S. Oueini, A. H. Nayfeh, and J. R. Pratt, Arch. Appl. Mech. 69, 585 (1999). 